\title{
Oscillatory positive expiratory pressure treatment in lower respiratory tract infection
}

\author{
YINGMENG NI, LIN DING, YOUCHAO YU, RANRAN DAI, HONG CHEN and GUOCHAO SHI \\ Department of Pulmonary and Critical Care Medicine, Ruijin Hospital, \\ Shanghai Jiao Tong University School of Medicine, Shanghai 200025, P.R. China
}

Received March 13, 2018; Accepted July 2, 2018

DOI: $10.3892 /$ etm.2018.6552

\begin{abstract}
Oscillatory positive expiratory pressure (OPEP) devices have been utilized as an adjunct therapy to conventional chest physiotherapy (CPT) to promote the clearance of respiratory secretions in individuals with impaired ability to cough, particularly in chronic diseases. However, few studies have focused on the effectiveness of OPEP in lower respiratory tract infection. In the present study, all patients with lower respiratory tract infections hospitalized in the Department of Pulmonary and Critical Care Medicine, Ruijin Hospital (Shanghai, China) between February 2016 and July 2017 were analyzed. Daily sputum quantity and purulence were recorded on the first 7 days of physiotherapy. Oxygenation index, partial pressure carbon dioxide, white blood cell count, neutrophil percentage, $\mathrm{C}$ reactive protein (CRP) and procalcitonin (PCT) levels before and after CPT were compared between patients who received OPEP and patients who received mechanical percussion (MP). Sputum was collected prior to and following CPT. A total of 17 patients received OPEP, while 10 received MP. The OPEP group exhibited improved postural drainage compared with the MP group after 7 days of physiotherapy. After 7 days of CPT, patients who received OPEP also exhibited a significantly improved oxygenation index, while the oxygenation index in the MP group did not improve. The improvement of partial pressure carbon dioxide was not significantly different between groups. The OPEP group also exhibited a greater decrease in white blood cell count,
\end{abstract}

Correspondence to: Dr Hong Chen or Dr Guochao Shi, Department of Pulmonary and Critical Care Medicine, Ruijin Hospital, Shanghai Jiao Tong University School of Medicine, 197 Ruijin Er Road, Shanghai 200025, P.R. China

E-mail: chenjiangjenny@sina.com

E-mail: shiguochao@hotmail.com

Abbreviations: ABG, arterial blood gas; CPT, chest physiotherapy; CRP, C reactive protein; OPEP, oscillatory positive expiratory pressure; $\mathrm{PCT}$, procalcitonin; $\mathrm{PEP}$, positive expiratory pressure; MP, mechanical percussion; WBC, white blood cell count

Key words: chest physiotherapy, oscillatory positive expiratory pressure, lower respiratory tract infection neutrophil percentage and CRP levels, compared with the MP group. However, the decrease in PCT level was similar in the OPEP and MP groups. Sputum culture results revealed that the rate of negative conversion was very low in both groups. There was no difference between the two groups in terms of hospitalization outcomes. In conclusion, OPEP exhibited a greater effectiveness in draining sputum, improving oxygenation and reducing inflammatory status in patients with lower respiratory tract infections compared with MP; however, it did not promote the elimination of microbes.

\section{Introduction}

Chest physiotherapy (CPT) is commonly used to improve the mobilization and removal of airway secretions in patients with respiratory dysfunction (1). In recent years, oscillatory positive expiratory pressure (OPEP) devices have been used to assist airway clearance (2).

OPEP therapy was first developed and described in Switzerland, as an adjunct or supplement to traditional airway clearance methods (2). The theoretical benefit of positive expiratory pressure (PEP) therapy is the ability to enhance and promote mucus clearance by either preventing airway collapse by stenting the airways (3), or increasing intrathoracic pressure distal to retained secretions by collateral ventilation, or by increasing functional residual capacity (4). OPEP combines the benefits previously described for PEP with airway vibrations or oscillations, which has been described as a 2-fold effect in airway clearance (5).

Konstan et al (6) demonstrated that OPEP was more effective compared with conventional techniques in clearing mucus from the airways of patients with cystic fibrosis. Girard and Terki (7) found significant improvements in forced expiratory volume in one second, forced vital capacity and peak expiratory flow with daily OPEP use in patients with asthma. OPEP was also demonstrated to be effective in clearing mucus from the airways in panbronchiolitis (8). The clinical effectiveness and high level of acceptance by patients make the OPEP devices widely popular (9).

However, the use of OPEP devices remains limited in chronic airway diseases including cystic fibrosis, bronchiectasis, bronchitis, bronchial asthma and primary ciliary dyskinesia syndrome. Few studies have focused on the effectiveness of OPEP in lower respiratory tract infection $(10,11)$. 
Another CPT method, the mechanical percussion (MP), is more frequently used in patients with lower respiratory tract infections (12). Mechanical chest percussors are machines constructed to imitate the manual clapping of the therapist. Ideally, these machines allow individual setting of frequency and intensity of percussion and are stable and lightweight for use by the patient him/herself (12). The therapeutic efficacy of these machines is not superior to a well-administered manual treatment (13); however, they relieve some of the physical strain of a treatment session. Another advantage of mechanical percussors is their potential use for self-administered therapy (12).

In the present study, the effectiveness of the use of OPEP was evaluated in patients with lower respiratory tract infection in the Respiratory Intensive Care Unit of Ruijin Hospital (Shanghai, China).

\section{Materials and methods}

Patients and physiotherapy methods. The present study was performed in the Respiratory Intensive Care Unit of Ruijin Hospital, ranked 4th in China, according to the China Hospital Ranking from the Hospital Management Institute of Fudan University (http://rank.cn-healthcare. $\mathrm{com} / \mathrm{rank} /$ profession-total-best). Ethical approval was received from the Ethics Committee of Ruijin Hospital to protect the confidentiality of patients and service user information and to enable appropriate information sharing. Full ethical approval was not required as all data were routinely recorded as part of standard clinical care.

The present study retrospectively reviewed electronic medical records of all patients from February 2016 to July 2017. Patients were selected if the diagnosis matched the following International Classification of Diseases, 10th Revision (ICD-10) codes for lower respiratory tract infection: Pneumonia (J12-18), acute bronchitis (J20), acute bronchiolitis (J21), unspecified acute lower respiratory infection (J22), bronchitis, not specified as acute or chronic (J40), influenza (J10 and J11), other chronic obstructive pulmonary disease with acute lower respiratory tract infection/acute exacerbation, unspecified (J44), whooping cough (A37), Legionnaires' disease (A48) or Chlamydia psittaci infection (A70), and were treated with MP or OPEP. Patients were excluded if they were not tolerant for at least 7 days of CPT or if they were not able to correctly independently use OPEP device.

CPT was initiated when patients were clinically stable. Patients were considered as clinically stable when the following criteria were met: i) Did not need invasive ventilation; ii) use time $<12 \mathrm{~h} /$ day if noninvasive ventilation was needed; iii) vasoactive drugs were not required; iv) arterial $\mathrm{pH}$ $>7.25$; v) body temperature $<37.5^{\circ} \mathrm{C}$.

The mechanical chest percussion was also initiated when patients were clinically stable. The mechanical chest percussor (G5 ${ }^{\circledR}$ Therassist $^{\circledR}$; Mettler Electronics Corp., Anaheim, CA, USA) was used to perform mechanical percussion for $10 \mathrm{~min}$ 4 times a day. A nurse was responsible for the correct use and good tolerance.

The Acapella ${ }^{\circledR}$ OPEP device (Green-DH model; Smiths Medical International, Ltd., Minneapolis, MN, USA) was used. This device uses a counterweighted plug and a magnet
Table I. Baseline characteristics of patients.

\begin{tabular}{lccc}
\hline Characteristics & MP & OPEP & P-value \\
\hline $\mathrm{n}$ & 10 & 17 & \\
Age (years) & $73 \pm 11$ & $68 \pm 14$ & 0.287 \\
Female (n) & 4 & 7 & 0.952 \\
APACHE II score & $17 \pm 6$ & $13 \pm 2$ & 0.057 \\
Oxygenation index & $236 \pm 82$ & $223 \pm 86$ & 0.719 \\
(mmHg) & & & \\
Arterial pH & $7.40 \pm 1.10$ & $7.39 \pm 0.06$ & 0.576 \\
PaCO $(\mathrm{kPa})$ & $6.11 \pm 1.80$ & $7.49 \pm 1.17$ & 0.048 \\
WBC (x109/1) & $7.89 \pm 2.21$ & $10.89 \pm 4.25$ & 0.051 \\
Neutrophil (\%) & $77.57 \pm 7.26$ & $79.81 \pm 9.26$ & 0.522 \\
CRP (mg/l) & $4.45 \pm 5.21$ & $3.54 \pm 3.62$ & 0.605 \\
PCT (pg/ml) & $0.22 \pm 0.28$ & $0.73 \pm 1.43$ & 0.282 \\
Diagnosis (n) & & & 0.698 \\
Severe pneumonia & 6 & 8 & \\
AECOPD & 2 & 4 & \\
Bronchiectasis & 0 & 2 & \\
$\quad$ Other & \\
Mechanical ventilation (n) & 8 & 10 & 0.482 \\
$\quad$ Noninvasive & 4 & 5 & \\
$\quad$ Invasive & 4 & 5 & \\
Duration of invasive & $12 \pm 2$ & $13 \pm 5$ & 0.796 \\
mechanical ventilation & & & \\
(days) & & & \\
\hline
\end{tabular}

${ }^{a} \mathrm{MP}$ group, one lower respiratory tract infection comorbid with diffuse panbronchiolitis and one lower respiratory tract infection comorbid with motorneuron disease; OPEP group, one lower respiratory tract infection comorbid with idiopathic pulmonary fibrosis and two lower respiratory tract infections comorbid with diffuse panbronchiolitis. MP, mechanical percussion; OPEP, oscillatory positive expiratory pressure; AECOPD, acute exacerbation of chronic obstructive pulmonary disease; APACHE, Acute Physiology and Chronic Health Evaluation; WBC, white blood cell count; CRP, C reactive protein; $\mathrm{PCT}$, procalcitonin; $\mathrm{n}$, number; $\mathrm{PaCO}_{2}$, partial pressure carbon dioxide.

to create airflow oscillations during expiratory flow. Patients were instructed to use the Acapella device a minimum of 5 times a day for at least $5 \mathrm{~min}$. As the tolerance of the patients increased, the PEP was gradually increased (14).

Measurements and outcomes. Patient baseline demographics and physiological characteristics were recorded. The daily sputum quantity and purulence were recorded on the first 7 days of CPT. Mucus sputum, mucopurulent sputum and purulent sputum were scored as 1,2 , and 3 respectively. $C$ reactive protein (CRP), procalcitonin (PCT), oxygenation index, arterial blood gas (ABG) analysis [including partial pressure ( $\mathrm{Pa}) \mathrm{O}_{2}, \mathrm{PaCO}_{2}$ and $\left.\mathrm{pH}\right]$, white blood cell count (WBC) and neutrophil percentage were performed by the hospital and recorded on 1 day before and 7 days after physiotherapy. The pre- and post-treatment differences were compared between the MP and OPEP groups. Sputum culture results before 

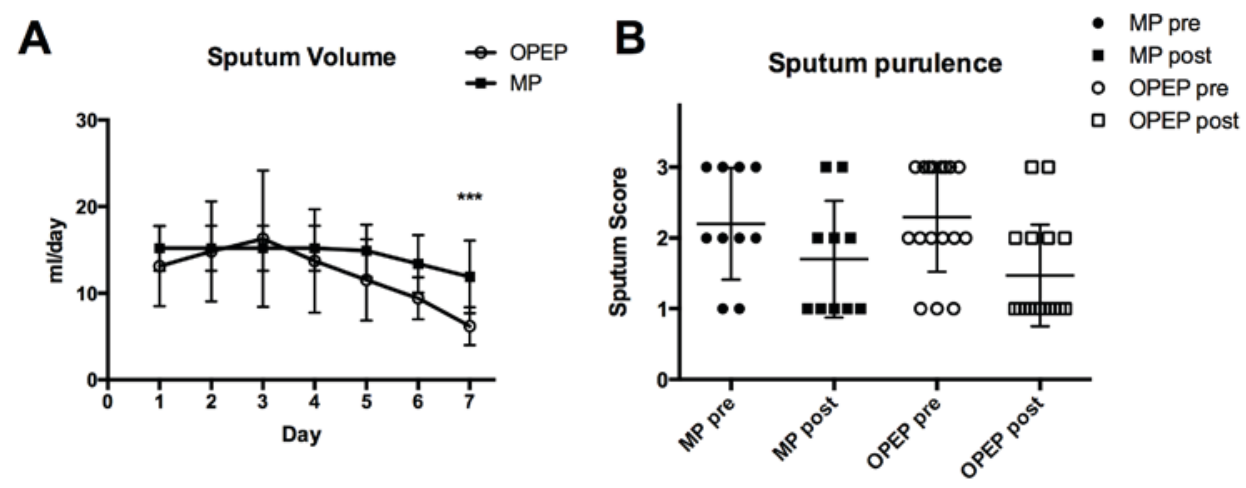

Figure 1. Daily sputum volume and purulence. (A) Daily sputum volume through the first 7 days of CPT. (B) Sputum purulence score before and 7 days after CPT. Mucus sputum, mucopurulent sputum and purulent sputum were scored as 1,2 , and 3 respectively. ${ }^{* * *} \mathrm{P}<0.001$ vs. the OPEP group. MP, mechanical percussion; OPEP, oscillatory positive expiratory pressure; CPT, chest physiotherapy; pre, before CPT; post, after CPT.

and $\geq 7$ days after physiotherapy were collected. Outcomes regarding hospitalization, including mortality, use of mechanical ventilation among survivors, stay in intensive care among survivors, and the length of hospitalization among survivors were also compared between the MP and OPEP groups.

Statistical analysis. Continuous variable data, including age, Acute Physiology and Chronic Health Evaluation (APACHE) II score, oxygenation index, arterial $\mathrm{pH}, \mathrm{PaCO}_{2}, \mathrm{WBC}$, neutrophil percentage, CRP, PCT, duration of invasive mechanical ventilation, duration of hospital stay, stay in intensive care and length of hospitalization among survivors are presented as the mean \pm standard deviation. Categorical variable data, including sex, diagnosis and mechanical ventilation, are presented as numbers. Sex, diagnosis, mechanical ventilation strategy, mortality during hospital stay, intensive care unit mortality and the sputum scores were compared between treatments groups using a $\chi^{2}$ test or a Fisher's exact test, as appropriate. Normally distributed data, including age, APACHE II score, oxygenation index, arterial $\mathrm{pH}, \mathrm{PaCO}_{2}, \mathrm{WBC}$ and neutrophil percentage were analyzed using a Student's t-test, and non-normally distributed data, including CRP and PCT were analyzed using a Mann-Whitney U test. $\mathrm{P}<0.05$ was considered to indicate a statistically significant difference. Analyses were performed using SPSS software (v.20.0; IBM Corp., Armonk, NY, USA).

\section{Results}

A total of 86 patients with lower respiratory tract infections were hospitalized in the Department of Pulmonary and Critical Care Medicine, Ruijin Hospital between February 2016 and July 2017, 17 of whom received OPEP and 10 received MP. The baseline characteristics of the 27 patients enrolled in the present study, including diagnosis, and mechanical ventilation method and duration are presented in Table I.

The APACHE II score, oxygenation index, arterial $\mathrm{pH}$, WBC, neutrophil percentage, and CRP and PCT levels were all similar in the OPEP and MO groups at baseline, while $\mathrm{PaCO}_{2}$ was significantly higher in the OPEP group $(\mathrm{P}=0.048$; Table I). The majority of patients were diagnosed with severe pneumonia without chronic respiratory disease. A total of 2 patients in the MP group and 4 in the OPEP group were
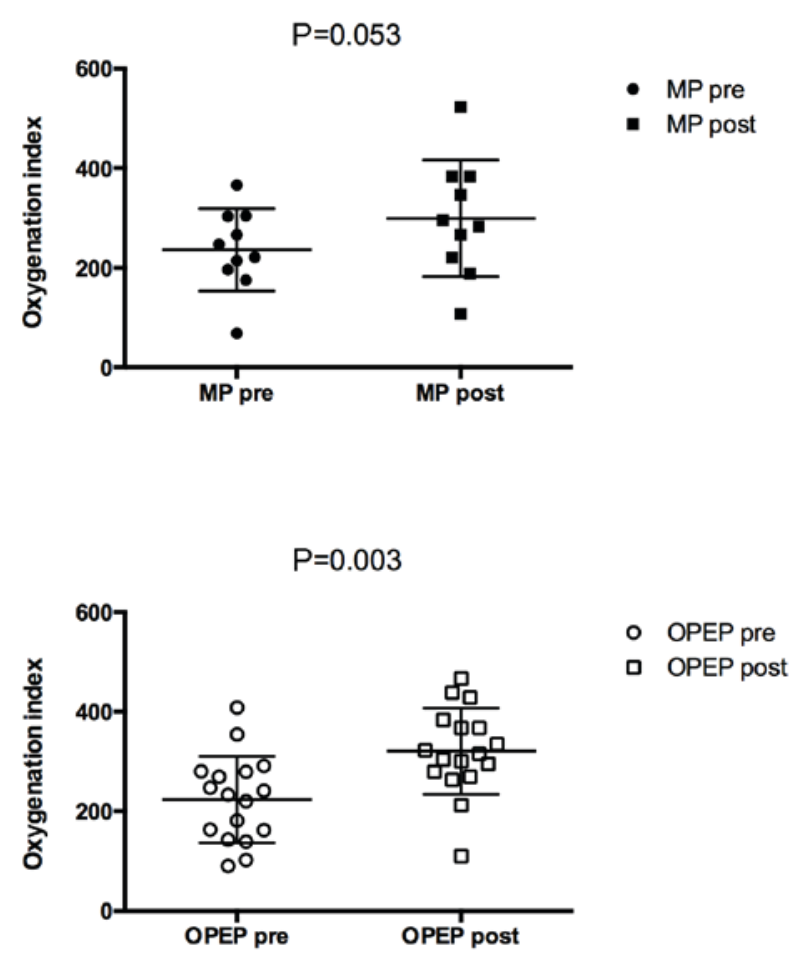

Figure 2. Oxygenation index prior to and following CPT. MP, mechanical percussion; OPEP, oscillatory positive expiratory pressure; pre, before CPT; post, after CPT; CPT, chest physiotherapy.

diagnosed with acute exacerbation of chronic obstructive pulmonary disease, and 2 patients in the OPEP group were diagnosed with bronchiectasis, which may explain the increased $\mathrm{PaCO}_{2}$ level in the OPEP group. A total of 4 patients in the MP group and 5 patients in the OPEP group were incubated and ventilated during hospitalization. The duration of invasive ventilation was similar between the groups. A total of 4 patients in the MP group and 5 patients in the OPEP group underwent non-invasive ventilation.

The daily sputum quantities were presented in Fig. 1A. Sputum quantities in the MP group declined gradually during the first 7 days of physiotherapy, while that in the OPEP group peaked after 2-3 days of OPEP, then sharply decreased. 
Table II. Measurements before and after chest physiotherapy.

\begin{tabular}{|c|c|c|c|c|c|c|}
\hline \multirow[b]{2}{*}{ Measurement } & \multicolumn{2}{|c|}{ MP } & \multirow[b]{2}{*}{ P-value } & \multicolumn{2}{|c|}{ OPEP } & \multirow[b]{2}{*}{ P-value } \\
\hline & Pre & Post & & Pre & Post & \\
\hline Oxygenation index $(\mathrm{mmHg})$ & $236 \pm 82$ & $299 \pm 117$ & 0.053 & $223 \pm 86$ & $322 \pm 89$ & 0.003 \\
\hline Arterial pH & $7.40 \pm 0.10$ & $7.4 \pm 0.06$ & 0.884 & $7.39 \pm 0.06$ & $7.37 \pm 0.03$ & 0.305 \\
\hline $\mathrm{PaCO}_{2}(\mathrm{kPa})$ & $6.11 \pm 1.80$ & $6.10 \pm 1.57$ & 0.989 & $7.49 \pm 1.17$ & $7.33 \pm 0.85$ & 0.371 \\
\hline WBC (x109/1) & $7.89 \pm 2.21$ & $7.80 \pm 2.72$ & 0.915 & $10.89 \pm 4.25$ & $8.80 \pm 2.92$ & 0.003 \\
\hline Neutrophil (\%) & $77.57 \pm 7.26$ & $73.43 \pm 10.94$ & 0.159 & $79.81 \pm 9.26$ & $71.23 \pm 8.14$ & 0.001 \\
\hline $\mathrm{CRP}(\mathrm{mg} / \mathrm{l})$ & $4.45 \pm 5.21$ & $1.79 \pm 2.18$ & 0.189 & $3.54 \pm 3.62$ & $1.27 \pm 1.79$ & 0.047 \\
\hline PCT $(\mathrm{pg} / \mathrm{ml})$ & $0.22 \pm 0.28$ & $0.10 \pm 0.14$ & 0.138 & $0.73 \pm 1.43$ & $0.08 \pm 0.12$ & 0.095 \\
\hline
\end{tabular}

WBC, white blood cell count; $\mathrm{CRP}, \mathrm{C}$ reactive protein; $\mathrm{PCT}$, procalcitonin; $\mathrm{PaCO}_{2}$, partial pressure carbon dioxide; $\mathrm{MP}$, mechanical percussion; OPEP, oscillatory positive expiratory pressure.

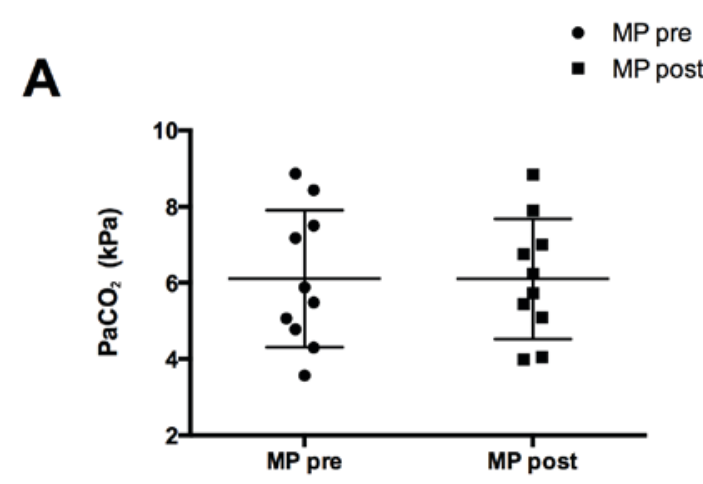

- OPEP pre

口 OPEP post
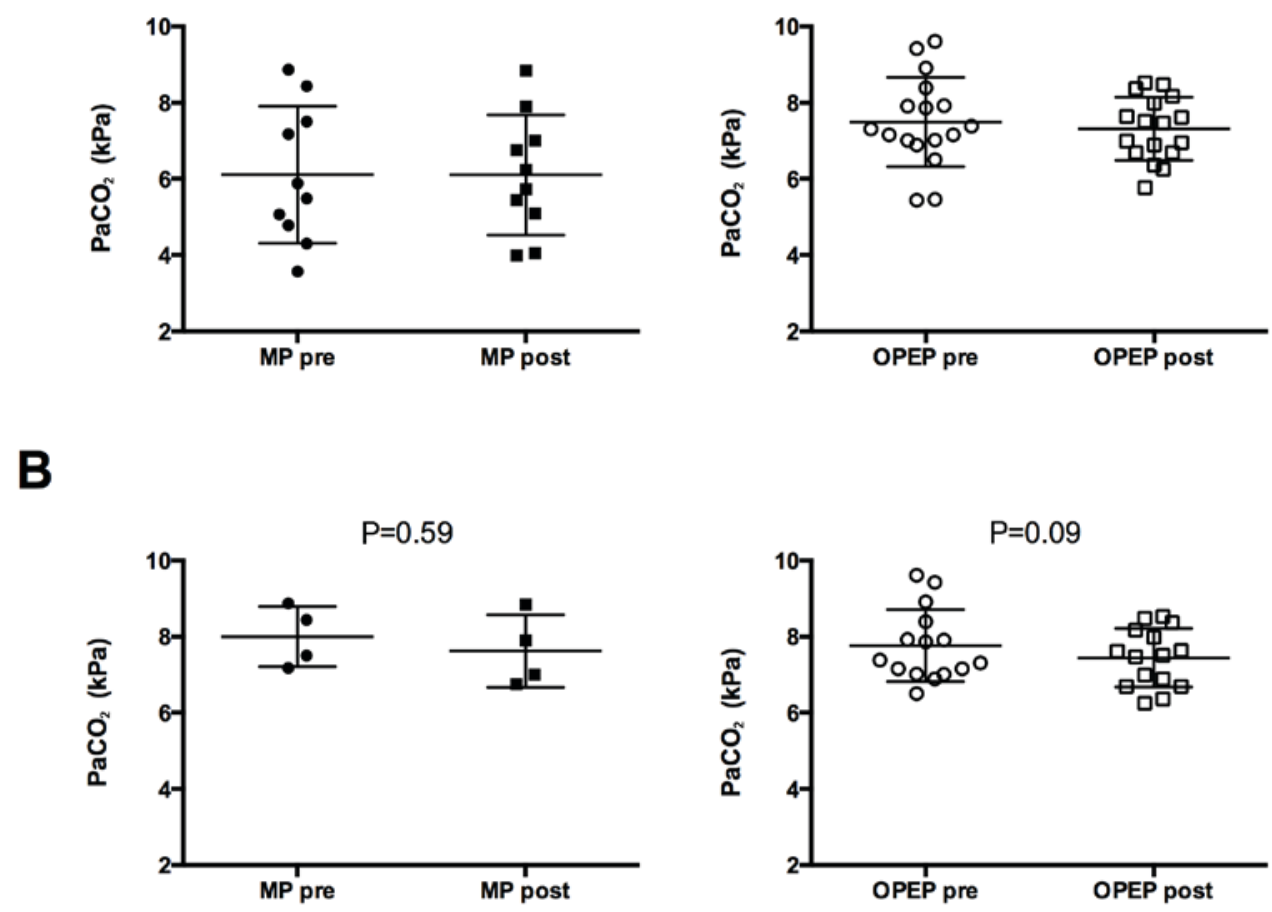

Figure 3. $\mathrm{PaCO}_{2}$ before and after CPT. (A) $\mathrm{PaCO}_{2}$ before and after $\mathrm{CPT}$ of all patients enrolled. (B) $\mathrm{PaCO}_{2}$ before and after $\mathrm{CPT}$ of patients with a PaCO $\mathrm{C}_{2}$ level higher than the normal reference range $(4.67-6.00 \mathrm{kPa})$. MP, mechanical percussion; OPEP, oscillatory positive expiratory pressure; $\mathrm{PaCO}_{2}$, partial pressure carbon dioxide; pre, before CPT; post, after CPT; CPT, chest physiotherapy.

On day 7 , the sputum quantity of the OPEP group was significantly smaller compared with the MP group $(\mathrm{P}=0.001)$ Sputum purulence 7 days after CPT slightly improved in both groups (Fig. 1B).

After 7 days of CPT, the oxygenation index of patients in the OPEP group increased from $223 \pm 86$ to $322 \pm 89$ ( $\mathrm{P}=0.003)$, while that of the MP group, which had a similar level of oxygenation index at baseline, only increased to $299 \pm 117$ $\left(\mathrm{P}=0.053\right.$; Fig. 2 and Table II). However, $\mathrm{PaCO}_{2}$ levels did not alter significantly in either the MP or OPEP group following physiotherapy (Fig. 3A; Table II), even in patients with a baseline $\mathrm{PaCO}_{2}$ level higher than the normal reference range (4.67-6.00 kPa; Fig. 3B) (15).

The WBC declined from $10.89 \pm 4.25 \times 10^{9}$ to $8.80 \pm 2.92 \times 10^{9} / 1$ $(\mathrm{P}=0.003)$ in the OPEP group after CPT; however, it remained unaltered in the MP group $(\mathrm{P}=0.915$; Fig. 4; Table II). Consistently, the neutrophil percentage also significantly decreased in the OPEP group ( $\mathrm{P}=0.0002)$, but not in the MP group $(\mathrm{P}=0.159)$. CRP is an inflammatory biomarker (16) and the CRP level in the OPEP group decreased significantly after CPT compared with the baseline level ( $\mathrm{P}=0.047$; Fig. 5; Table II); no alteration was evident in the MP group. However, 

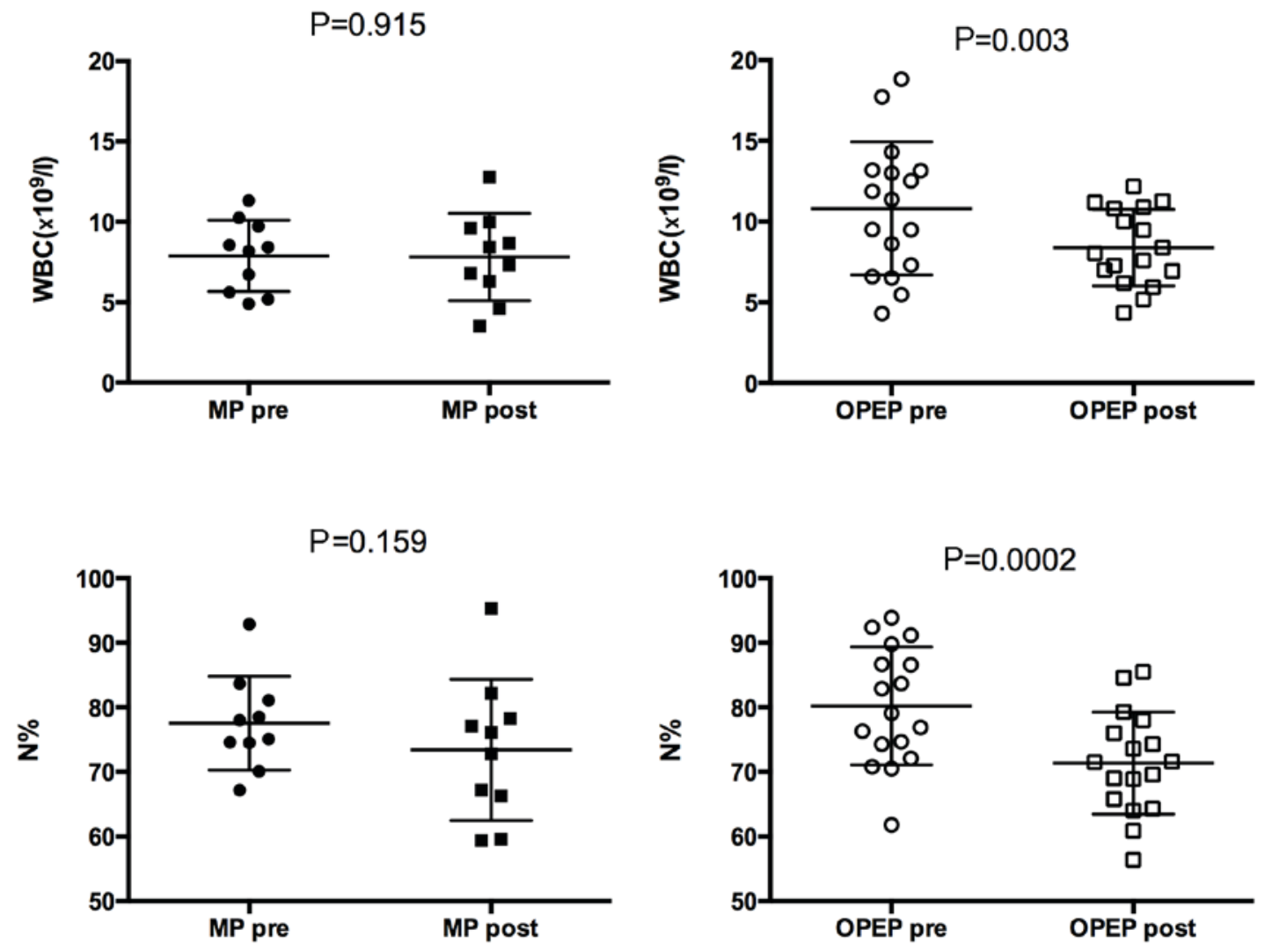

Figure 4. WBC and N\% prior to and following CPT. WBC, white blood cell count; pre, before CPT; post, after CPT; N\%, neutrophil percentage; CPT, chest physiotherapy.
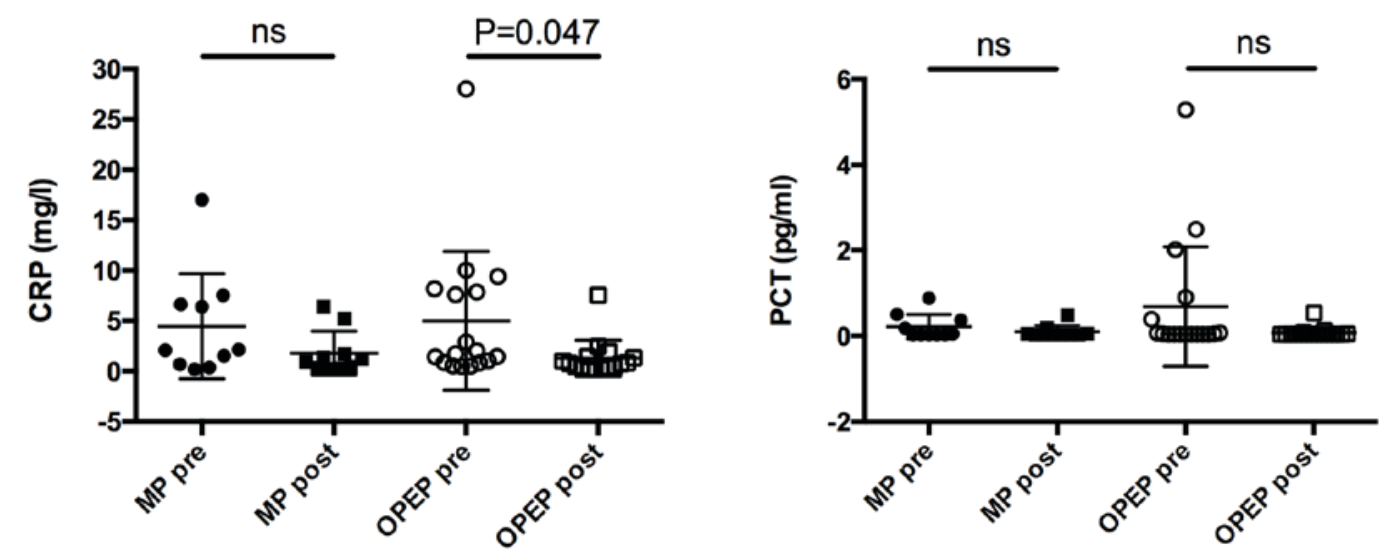

Figure 5. CRP and PCT levels before and after CPT. MP, mechanical percussion; OPEP, oscillatory positive expiratory pressure; pre, before CPT; post, after $\mathrm{CPT}$; ns, not significant; $\mathrm{CRP}, \mathrm{C}$ reactive protein; $\mathrm{PCT}$, procalcitonin; $\mathrm{CPT}$, chest physiotherapy.

the expression level of PCT, a biomarker of infection, did not alter significantly in either group (Fig. 5; Table II).

Sputum culture results demonstrated that the elimination of microbes was not associated with the method of CPT (Table III). The rate of sputum culture negative conversion was low in both the OPEP group (4/17) and the MP group (3/10). There was no difference in the duration of hospital stay, the rate of mortality in hospital, the duration of stay in intensive care among survivors or the length of hospitalization among survivors between the two groups (Table IV).

\section{Discussion}

CPT is considered a standard method used for the mobilization and removal of airway secretions in patients with respiratory dysfunction, particularly chronic lung disease including 
Table III. Sputum culture prior to and following chest physiotherapy.

A, Mechanical percussion

\begin{tabular}{cll}
\hline Patient & \multicolumn{1}{c}{ Pre } & \\
\hline 1 & Candida albicans & Post \\
2 & Candida tropicalis & Negative \\
3 & Acinetobacter baumannii & Candida tropicalis \\
4 & Pseudomonas aeruginosa & Acinetobacter baumannii \\
5 & Acinetobacter baumannii & Negative \\
6 & Negative & Acinetobacter baumannii \\
7 & Candida albicans & Negative \\
8 & Negative & Negative \\
9 & Pseudomonas aeruginosa & Negative \\
10 & Klebsiella pneumoniae & Pseudomonas aeruginosa \\
\hline
\end{tabular}

B, Oscillatory positive expiratory pressure

\begin{tabular}{|c|c|c|}
\hline Patient & Pre & Post \\
\hline 1 & Acinetobacter baumannii & Negative \\
\hline 2 & Pseudomonas aeruginosa & Pseudomonas aeruginosa, Klebsiella pneumoniae \\
\hline 3 & Negative & Negative \\
\hline 4 & Stenotrophomonas maltophilia & Klebsiella pneumoniae \\
\hline 5 & Pseudomonas aeruginosa & Pseudomonas aeruginosa \\
\hline 6 & Negative & Negative \\
\hline 7 & Pseudomonas aeruginosa & Negative \\
\hline 8 & Klebsiella pneumoniae & Klebsiella pneumonia, Acinetobacter baumannii \\
\hline 9 & Negative & Negative \\
\hline 10 & Enterococcus faecium & Klebsiella pneumonia \\
\hline 11 & Pseudomonas aeruginosa & Negative \\
\hline 12 & Negative & Negative \\
\hline 13 & Klebsiella pneumoniae & Klebsiella pneumoniae \\
\hline 14 & Negative & Negative \\
\hline 15 & Negative & Negative \\
\hline 16 & Klebsiella pneumoniae & Klebsiella pneumoniae \\
\hline 17 & Klebsiella pneumoniae & Negative \\
\hline
\end{tabular}

Table IV. Outcomes of hospitalization.

\begin{tabular}{|c|c|c|c|}
\hline Outcome & MP & OPEP & P-value \\
\hline Duration of chest physiotherapy (days) & $7 \pm 1$ & $8 \pm 1$ & 0.069 \\
\hline Duration of hospital stay (days) & $19 \pm 8$ & $24 \pm 13$ & 0.220 \\
\hline Mortality in hospital & 1 & 0 & 0.370 \\
\hline Mortality in intensive care unit & 1 & 0 & 0.370 \\
\hline Stay in intensive care, among survivors (days) & $20 \pm 8$ & $25 \pm 13$ & 0.209 \\
\hline Length of hospitalization, among survivors (days) & $22 \pm 8$ & $23 \pm 12$ & 0.220 \\
\hline
\end{tabular}

MP, mechanical percussion; OPEP, oscillatory positive expiratory pressure. 
cystic fibrosis, bronchiectasis, bronchitis, bronchial asthma and primary ciliary dyskinesia syndrome (1). The conventional regimen of CPT includes percussion, vibration and compression with or without postural drainage and assisted coughing (17). Airway clearance is achieved by postural drainage, manual chest percussion, vibration and compression, and directed coughing. Recently, on the basis of CPT, OPEP devices have been used to assist with airway clearance $(5,9)$.

OPEP is a combination of PEP therapy and oscillation. PEP works by shifting the equal pressure point peripherally to maximize airflow and to prevent dynamic airway collapse (9). This increases expiration time and alveolar ventilation, and facilitates gas exchange (9). Oscillations decrease the viscoelastic properties of mucus, making it easier to mobilize mucus in the airways, allowing short bursts of increased expiratory airflow that assist in mobilizing secretions up the airways (5).

Acapella and Flutter are the two most commonly used OPEP devices $(18,19)$. Volsko et al (18) found that Acapella and Flutter exhibit similar operating performance characteristics in terms of pressure amplitude and frequency. The performance of Acapella is not dependent on device and/or patient orientation and, therefore, it may be easier to use for certain patients, particularly those with lower expiratory flow (19). Acapella comes in 3 models: Green, for patients with expiratory flow $>15$ 1/min; blue, for patients with expiratory flow $<151 / \mathrm{min}$, and Choice, which can be disassembled into 4 easy to clean parts that can withstand autoclaving, boiling or dishwashing (20).

Previous studies have demonstrated the efficacy of OPEP in chronic diseases with impaired secretion clearance and expectoration $(6-8,19)$. However, to the best of the authors' knowledge, no cohort study focused on the efficacy of OPEP in respiratory infectious disease. The use of OPEP for patients with H1N1 was only reported in one case report (10). In the aforementioned case report it was shown that with the use of Acapella, the patient was easily able to clear airway secretions, and chest radiography revealed consistent daily improvement and resolution of signs of atelectasis within a week.

In the present study, a single center experience of the use of OPEP in lower respiratory tract infection was reported. Sputum quantity curve indicated that OPEP was more effective for sputum clearance compared with MP following 7 days of treatment. The effectiveness of OPEP in sputum clearance has been confirmed by numerous studies regarding OPEP in chronic airway diseases $(5$, 18-20). The present study indicated that OPEP also exhibited a similar effectiveness in acute respiratory infection.

OPEP is considered to be able to maximize airflow, increase expiratory time and alveolar ventilation, and facilitate gas exchange (9). However, in the present study, OPEP improved the oxygenation index but not the $\mathrm{PaCO}_{2}$ levels compared with the MP treatment. Considering that the majority of patients enrolled in the present study did not have chronic obstructive respiratory disease, the improvement in oxygenation index was likely due to the amelioration of inflammation status. The amelioration of inflammation status was indicated by the decreased WBC, neutrophil percentage and CRP levels in the OPEP group following physiotherapy compared with the MP group. However, OPEP was not more effective on PCT decrease or sputum culture negative conversion compared with MP, indicating that OPEP did not directly promote the elimination of microbes.
The limitations of the present study include its retrospective nature. All data were collected from electronic medical records. Oxygenation index, $\mathrm{ABG}, \mathrm{WBC}$ and neutrophil data were collected daily, while CRP, PCT and sputum culture data were not. The small sample size also limited the reliability of the present study and was primarily due to economic reasons, as Acapella is not covered by social health insurance, and is bought by the patients themselves. In spite of these limitations, this is the first study to investigate the efficacy of OPEP in lower respiratory tract infection. A prospective study with a larger sample size is required to validate these findings. Finally, the present study investigated the effectiveness of OPEP for short duration. Further studies are required to establish the long-term clinical effects of OPEP therapy.

Overall, OPEP exhibited a greater effectiveness compared with MP to drain sputum, improve oxygenation and reduce inflammation in patients with lower respiratory tract infection. However, it did not directly promote the elimination of microbes.

\section{Acknowledgements}

Not applicable.

\section{Funding}

The present study was supported by Shanghai Sailing Program (grant no. 16YF1406900).

\section{Availability of data and materials}

The datasets used and analyzed during the present study are available from the corresponding author on reasonable request.

\section{Authors' contributions}

YN, LD, RD and HC, contributed to the analysis of data, had full access to all of the data in the study and take responsibility for the integrity and accuracy of the data analysis. YY and GS contributed to analysis and interpretation of data and the preparation of the manuscript. YN served as principal author. All authors read and approved the final version of the manuscript.

\section{Ethics approval and consent to participate}

The ethics approval for protection of confidentiality of patients and service user information, and appropriate information sharing in the present study was received from the Ethics Committee of Ruijin Hospital (Shanghai, China). Full ethics approval was not required as all data were routinely recorded as part of standard clinical care.

\section{Patient consent for publication}

Not applicable.

\section{Competing interests}

The authors declare that they have no competing interests. 


\section{References}

1. Arens R, Gozal D, Omlin KJ, Vega J, Boyd KP, Keens TG and Woo MS: Comparison of high-frequency chest compression and conventional chest physiotherapy in hospitalized patients with cystic fibrosis. Am J Respir Crit Care Med 150: 1154-1157, 1994

2. Pryor JA: Physiotherapy for airway clearance in adults. Eur Respir J 14: 1418-1424, 1999.

3. Oberwaldner B, Evans JC and Zach MS: Forced expirations against a variable resistance: A new chest physiotherapy method in cystic fibrosis. Pediatr Pulmonol 2: 358-367, 1986.

4. Groth S, Stafanger G, Dirksen H, Andersen JB, Falk M and Kelstrup M: Positive expiratory pressure (PEP-mask) physiotherapy improves ventilation and reduces volume of trapped gas in cystic fibrosis. Bull Eur Physiopathol Respir 21: 339-343, 1985.

5. McIlwaine M: Physiotherapy and airway clearance techniques and devices. Paediatr Respir Rev 7 (Suppl 1): S220-S222, 2006.

6. Konstan MW, Stern RC and Doershuk CF: Efficacy of the Flutter device for airway mucus clearance in patients with cystic fibrosis J Pediatr 124: 689-693, 1994

7. Girard JP and Terki N: The Flutter VRP1: A new personal pocket therapeutic device used as an adjunct to drug therapy in the management of bronchial asthma. J Investig Allergol Clin Immunol 4: 23-27, 1994.

8. Burioka N, Sugimoto Y, Suyama H, Hori S, Chikumi H and Sasaki T: Clinical efficacy of the FLUTTER device for airway mucus clearance in patients with diffuse panbronchiolitis. Respirology 3: 183-186, 1998.

9. Nicolini A, Mascardi V, Grecchi B, Ferrari-Bravo M, Banfi P and Barlascini C: Comparison of effectiveness of temporary positive expiratory pressure versus oscillatory positive expiratory pressure in severe COPD patients. Clin Respir J 12: 1274-1282, 2018.

10. Narula D and Nangia V: Use of an oscillatory PEP device to enhance bronchial hygiene in a patient of post-H1NI pneumonia and acute respiratory distress syndrome with pneumothorax. BMJ Case Rep: 2014. pii: bcr2013202598, 2014.
11. Castro AA, Calil SR, Freitas SA, Oliveira AB and Porto EF: Chest physiotherapy effectiveness to reduce hospitalization and mechanical ventilation length of stay, pulmonary infection rate and mortality in ICU patients. Respir Med 107: 68-74, 2013.

12. Zach MS and Oberwaldner B: Chest physiotherapy-the mechanical approach to antiinfective therapy in cystic fibrosis. Infection 15: 381-384, 1987.

13. Maxwell M and Redmond A: Comparative trial of manual and mechanical percussion technique with gravity-assisted bronchial drainage in patients with cystic fibrosis. Arch Dis Child 54: 542-544, 1979.

14. Alves CE, Nunes LQ and Melo PL: Mechanical analysis of an oscillatory positive expiratory pressure device used in respiratory rehabilitation. Conf Proc IEEE Eng Med Biol Soc 2010: 2477-2480, 2010.

15. Carrera E, Kim DJ, Castellani G, Zweifel C, Smielewski P, Pickard JD and Czosnyka M: Effect of hyper- and hypocapnia on cerebral arterial compliance in normal subjects. J Neuroimaging 21: 121-125, 2011.

16. Wu Y, Potempa LA, El Kebir D and Filep JG: C-reactive protein and inflammation: Conformational changes affect function. Biol Chem 396: 1181-1197, 2015.

17. Newhouse PA, White F, Marks JH and Homnick DN: The intrapulmonary percussive ventilator and flutter device compared to standard chest physiotherapy in patients with cystic fibrosis. Clin Pediatr (Phila) 37: 427-432, 1998.

18. Volsko TA, DiFiore JM and Chatburn RL: Performance comparison of two oscillating positive expiratory ressure devices: Acapella versus flutter. Respir Care 48: 124-130, 2003.

19. Van Fleet H, Dunn DK, McNinch NL and Volsko TA: Evaluation of functional characteristics of 4 oscillatory positive pressure devices in a simulated cystic fibrosis model. Respir Care 62: 451-458, 2017.

20. Myers TR: Positive expiratory pressure and oscillatory positive expiratory pressure therapies. Respir Care 52: 1308-1327, 2007. 